\title{
Melatonin in chronic kidney disease: a promising chronotherapy targeting the intrarenal renin-angiotensin system
}

\author{
Asadur Rahman ${ }^{1}$ - Arif UI Hasan ${ }^{1} \cdot$ Hiroyuki Kobori ${ }^{1}$
}

Received: 18 December 2018 / Revised: 30 December 2018 / Accepted: 1 January 2019 / Published online: 13 February 2019

(c) The Japanese Society of Hypertension 2019

The renin-angiotensin system (RAS) plays a crucial role in the conservation of extracellular volume homeostasis and blood pressure through complex molecular mechanisms [1]. The augmented activity of circulating levels of RAS components significantly affects the pathogenesis of chronic kidney disease (CKD) and subsequently end-stage renal disease (ESRD). Apart from this well-known systemic RAS, existence of a local RAS has been reported in different tissues, including the kidneys [2]. Inappropriate activation of intrarenal RAS has been observed in a variety of animal models of renal disease as an initial response to hypoperfusion and is considered as an important enhancer of the progression of CKD [1]. The upregulation of multiple RAS components is usually detected in the absence of increased plasma renin activity or plasma angiotensin (Ang) II. Emerging evidence has suggested that enhanced activation of intrarenal RAS triggers increased reactive oxygen species (ROS) production, imbalanced sodium homeostasis, glomerular sclerosis, and tubulointerstitial fibrosis [3, 4]. Furthermore, elevation of the blood pressure (BP) is also evident in subjects with CKD. Disruption of the circadian rhythm of BP, particularly attenuation of the night-time drop in $\mathrm{BP}(<10 \%$ decline; referred as non-dipper), has also been associated with the aggravated intrarenal RAS in $\mathrm{CKD}$, and it is an important predictor of further progression of CKD to ESRD [5]. Additionally, sleep disorders are highly prevalent in CKD patients with additional morbidity and mortality burdens. Sleep disorders, especially obstructive sleep apnea, can contribute to CKD through a direct effect of intrarenal hypoxia on the kidney and activation of the intrarenal RAS (Fig. 1) [6].

Hiroyuki Kobori

hkobori@iuhw.ac.jp

1 Department of Pharmacology, School of Medicine, International University of Health and Welfare, 4-2 Kozunomori, Narita, Chiba 286-8686, Japan
Melatonin (N-acetyl-5-methoxytryptamine) is primarily secreted from the pineal gland under the influence of light/ dark cycle in vertebrates [7]. Light stimulus is sensed by the ocular photoreceptors in the retina and then is transmitted to the suprachiasmatic nucleus ( $\mathrm{SCN}$ ) in the hypothalamus and finally to the pineal gland. Melatonin production is suppressed by light, while it is secreted predominantly at night. It influences circadian rhythmicity by interacting with the master clock in the SCN, as well as with peripheral clocks in different organs, including the kidneys, thereby regulating numerous biological functions [5]. Furthermore, melatonin exerts pluripotent protective effects in the kidneys by receptor-mediated or receptor-independent biological actions [8]. It modulates mitochondrial metabolism, increases ATP production and protects mitochondria against nitrative damage. Melatonin limits oxidative stress directly by inactivation of free radicals through the donation of one or more electrons. Additionally, pineal indoleamine, a melatonin metabolite, exhibits indirect antioxidant action by stimulating the expression of major antioxidant enzymes, for instance, superoxide dismutase and catalases. In chronic injury, melatonin played an important role by suppressing pro-inflammatory mediators and initiating a protective effect against inflammatory damage [8]. Moreover, melatonin plays a critical role in maintaining the bioavailability of nitric oxide (believe to decrease in hypertension) by acting on melatonin receptor MT2, thus promoting endothelium-dependent vasorelaxation in the mesenteric arteries (MAs) of spontaneously hypertensive rats (SHRs) [9]. Long-term treatment with melatonin increased the expression of markers associated with vasoprotection and decreased oxidative stress and inflammation in mice [10]. Other than these pleotropic effects, melatonin is an important regulator of the circadian sleep-wake cycle since levels of melatonin peak at night and gradually decrease during the daytime in healthy subjects. In contrast, circulating melatonin levels are decreased in elderly subjects; additionally, the acrophase of melatonin is altered in different 
Fig. 1 In subjects with chronic kidney disease (CKD), nighttime secretion of melatonin is significantly impaired. The alerted melatonin acrophase is then associated with the aggravated intrarenal renin-angiotensin system (RAS), subsequently causing increased reactive oxygen species (ROS) production, sodium retention, inflammation, and fibrosis. These pathological changes in the kidneys trigger an increase in blood pressure (BP), as well as altering the dipping pattern of BP from dipper to non-dipper type. Disrupted melatonin acrophase and activated intrarenal RAS are also associated with sleep disorders. All of these pathologies enhance the progression of CKD to endstage renal disease (ESRD).

However, exogenous melatonin reverses all of these pathological changes in the kidneys; subsequently, a slower progression of CKD with better quality of life would be attained by not only reducing BP but also normalizing the dipping pattern and improving sleep conditions

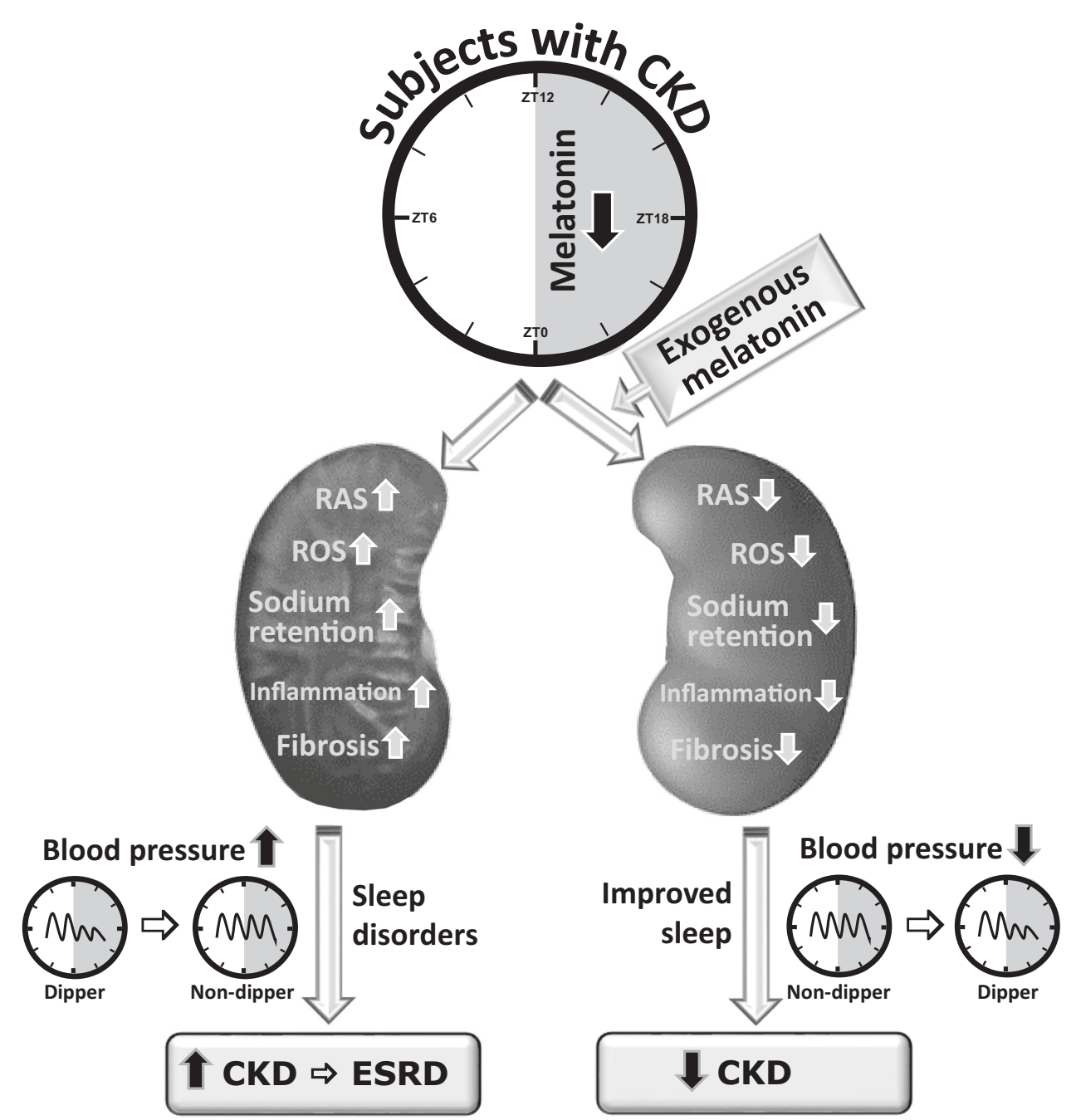

pathological conditions, such as the non-dipper type of hypertension in CKD [7].

In $\mathrm{CKD}$, renal function progressively declines due to a reduction in the number of functional nephrons. Irrespective of the initial cause of injury, CKD involves persistent inflammation, necrosis, and apoptosis, leading to the tubular atrophy, glomerulosclerosis, and tubulointerstitial fibrosis $[7,8]$. Consequently, these renal pathologies are associated with proteinuria, which is not only an indicator of renal damage but also represents a risk factor for the progression of CKD to ESRD. Therefore, therapeutic strategies for CKD should be designed to suppress the aforementioned pathologies. Moreover, KDIGO clinical practice guidelines include the close monitoring and appropriate treatment of high BP in subjects with CKD [11]. Since the systemic and intrarenal RAS are highly activated in subjects with CKD, agents interfering with the RAS are considered a cornerstone of CKD treatment. Our group demonstrated that intervention with an angiotensin II type 1 receptor blocker, with or without hydrochlorothiazide, improved nocturnal hypertension and slowed the progression of renal injury in patients with CKD to ESRD with cardiovascular complications [5]. However, owing to the partial benefits or limitations of the current and available therapeutics, novel agents would be explored in terms of reducing the burden or progression of $\mathrm{CKD}$, as well as improving the quality of life.

In the current issue of Hypertension Research, Ohashi et al. [12] have summarized their works and the previously reported investigations regarding the contribution of melatonin to attenuating the CKD. The renoprotective effects of melatonin in a variety of renal injury animal models, including 5/6 nephrectomy, unilateral ureteral obstruction, spontaneously hypertensive rats and adriamycin inducednephropathy, have been shown by suppressing oxidative stress, renal inflammation, and renal damage. It has also been revealed that impaired night-time melatonin secretion correlates negatively with that of urinary angiotensinogen (AGT), a surrogate marker for the intrarenal RAS [2]. Most strikingly Ishigaki and Ohashi et al. [13] demonstrated that melatonin administration in 5/6 nephrectomized rats caused a significant reduction in the levels of urinary AGT. 
Moreover, melatonin treatment attenuated the expression of AGT protein in the kidney, compared with that in untreated nephrectomized rats. Consistently, the expression of AT1 receptor and Ang II in the kidney was attenuated in melatonin-treated nephrectomized rats, suggesting a critical role of melatonin in suppressing the intrarenal RAS. Since the RAS is interconnected with ROS, inflammation, and fibrosis, melatonin treatment is believed to suppress all of these pathological changes in the kidneys and result in slower progression of CKD.

The protective effects of melatonin have also been demonstrated in several models of hypertension-mediated nephropathy. Specifically, in the two-kidney one-clip (2K1C) renovascular hypertension model (in which the intrarenal RAS is highly aggravated), melatonin treatment reduced $\mathrm{BP}$, as well as prevented the elevation of the levels of malondialdehyde (MDA) and myeloperoxidase (MPO), which are markers for oxidative stress [14]. Similarly, the intrarenal RAS is also known to be activated in a high salt diet-induced hypertension model in Dahl salt-sensitive rats, in which melatonin significantly reduced BP and kidney injury via a direct antioxidant effect [15]. These observations are consistent with the findings of Simko et al., [16] who showed that melatonin exerts antihypertensive action in N(G)-nitro-L-arginine-methyl ester (L-NAME)-induced hypertension in rats without affecting the systemic RAS. In clinical settings, Ishigaki et al. [17] demonstrated that impaired night-time melatonin secretion was associated with night-time intrarenal RAS activation, which might be involved in the underlying pathogenesis of progressive CKD. Conversely, bedtime intake of melatonin or the addition of melatonin to antihypertensive therapy is effective in ameliorating the non-dipping type of hypertension, a frequently observed phenomenon in CKD [5].

Melatonin is known to play an important role in initiating and maintaining sleep. Accumulating evidence has suggested that subjects with CKD or on dialysis exhibit altered circadian rhythms of melatonin levels in the blood, and the production of melatonin is decreased with the progression of CKD to ESRD [6]. It has been shown that both slow wave sleep and rapid eye movement sleep were significantly increased during dark periods in rats with subtotal nephrectomy, compared with the sleep patterns of control rats [5]. The EMSCAP study demonstrated that melatonin intervention improved objective sleep onset latency, sleep time and efficiency, reduced sleep fragmentation and improved subjective sleep parameters, along with the normalization of nocturnal melatonin surges in dialysis patients [8]. In fact, obstructive sleep apnea has been shown to be triggered by activation of the intrarenal RAS; [6] therefore, it might be stated that melatonin can improve obstructive sleep apnea by attenuating the intrarenal RAS and could assist in providing better quality of life for CKD patients undergoing hemodialysis.

In numerous experimental animal models of CKD and clinical settings, the effects of melatonin have been obvious on the improvement of renal function and lowering of BP, accompanied by the normalization of circadian rhythms, i.e., non-dipper to dipper type of BP variation and improvement of sleep disorders. These pleiotropic and protective effects of melatonin are the consequences of numerous complementary interconnecting mechanisms, including the reduction of oxidative stress, inflammation and fibrosis. Additionally, Ohashi and colleagues [12] showed the crucial role of melatonin in the suppression of the intrarenal RAS, which is believed to be involved in the slower progression of kidney injury, accompanied by increased sodium excretion, reduced BP and restoration of nocturnal BP dipping in subjects with CKD (Fig. 1). Considering the numerous benefits of melatonin, large clinical trials in phases 2 and 3 have been under way regarding the improvement of quality of life in patients with CKD and ESRD who have sleep disorders and are undergoing dialysis (ClinicalTrials.gov; NCT01922999, NCT00698360, NCT00388661). The outstanding renoprotective findings in clinical settings with melatonin might open a new window into therapeutic strategies for subjects with CKD.

\section{Compliance with ethical standards}

Conflict of interest The authors declare no conflicts of interest.

Publisher's note: Springer Nature remains neutral with regard to jurisdictional claims in published maps and institutional affiliations.

\section{References}

1. Kobori H, Urushihara M. Augmented intrarenal and urinary angiotensinogen in hypertension and chronic kidney disease. Pflüg Arch. 2012;465:3-12.

2. Kobori H, Nangaku M, Navar LG, Nishiyama A. The intrarenal renin-angiotensin system: from physiology to the pathobiology of hypertension and kidney disease. Pharmacol Rev. 2007;59:251-87.

3. Kobori H, Nishiyama A. Effects of tempol on renal angiotensinogen production in Dahl salt-sensitive rats. Biochem Biophys Res Commun. 2004;315:746-50.

4. Navar LG. Intrarenal renin-angiotensin system in regulation of glomerular function. Curr Opin Nephrol Hypertens. 2014;23:38-45.

5. Rahman A, Hasan AU, Nishiyama A, Kobori H. Altered circadian timing system-mediated non-dipping pattern of blood pressure and associated cardiovascular disorders in metabolic and kidney diseases. Int J Mol Sci. 2018;19:E400. pii

6. Nicholl DD, Hanly PJ, Poulin MJ, Handley GB, Hemmelgarn BR, Sola DY, et al. Evaluation of continuous positive airway pressure 
therapy on renin-angiotensin system activity in obstructive sleep apnea. Am J Respir Crit Care Med. 2014;190:572-80.

7. Russcher M, Koch B, Nagtegaal E, Van Der Putten K, Ter Wee P, Gaillard C. The role of Melatonin Treatment in chronic kidney disease. Front Biosci. 2012;17:2644-56.

8. Hrenak J, Paulis L, Repova K, Aziriova S, Nagtegaal EJ, Reiter $\mathrm{RJ}$, et al. Melatonin and renal protection: novel perspectives from animal experiments and human studies (review). Curr Pharm Des. 2015;21:936-49.

9. Qiu F, Liu X, Zhang Y, Wu Y, Xiao D, Shi L. Aerobic exercise enhanced endothelium-dependent vasorelaxation in mesenteric arteries in spontaneously hypertensive rats: the role of melatonin. Hypertens Res. 2018;41:718-29.

10. Agabiti-Rosei C, Favero G, De Ciuceis C, Rossini C, Porteri E, Rodella LF, et al. Effect of long-term treatment with melatonin on vascular markers of oxidative stress/inflammation and on the anticontractile activity of perivascular fat in aging mice. Hypertens Res. 2017;40:41-50.

11. National Kidney Foundation. KDIGO Clinical Practice Guideline for the Management of Blood Pressure in Chronic Kidney Disease. Kidney Int Suppl. 2012;2:383-93.

12. Ohashi $\mathrm{N}$, Ishigaki $\mathrm{S}$, Isobe $\mathrm{S}$. The pivotal role of melatonin in ameliorating chronic kidney disease by suppression of the renin- angiotensin system in the kidney. Hypertens Res. 2019. https:// doi.org/10.1038/s41440-018-0186-2.

13. Ishigaki S, Ohashi $\mathrm{N}$, Matsuyama $\mathrm{T}$, Isobe $\mathrm{S}$, Tsuji $\mathrm{N}$, Iwakura $\mathrm{T}$, et al. Melatonin ameliorates intrarenal renin-angiotensin system in a 5/6 nephrectomy rat model. Clin Exp Nephrol. 2018;22:539-49.

14. Erşahin M, Sehirli O, Toklu HZ, Süleymanoglu S, EmekliAlturfan E, Yarat A, et al. Melatonin improves cardiovascular function and ameliorates renal, cardiac and cerebral damage in rats with renovascular hypertension. J Pineal Res. 2009;47: 97-106.

15. Leibowitz A, Volkov A, Voloshin K, Shemesh C, Barshack I, Grossman E. Melatonin prevents kidney injury in a high salt dietinduced hypertension model by decreasing oxidative stress. J Pineal Res. 2016;60:48-54.

16. Simko F, Baka T, Krajcirovicova K, Repova K, Aziriova S, Zorad $\mathrm{S}$, et al. Effect of Melatonin on the Renin-AngiotensinAldosterone System in 1-NAME-Induced Hypertension. Molecules. 2018;23:E265. pii

17. Ishigaki $\mathrm{S}$, Ohashi $\mathrm{N}$, Isobe $\mathrm{S}$, Tsuji $\mathrm{N}$, Iwakura $\mathrm{T}$, Ono $\mathrm{M}$, et al. Impaired endogenous night-time melatonin secretion relates to intrarenal renin-angiotensin system activation and renal damage in patients with chronic kidney disease. Clin Exp Nephrol. $2016 ; 20: 878-84$ 